\title{
Knowledge Sharing for Strengthening Manufacturing Operation and Business Performance
}

\author{
T.T. Huang ${ }^{1}$, R. A. Stewart ${ }^{1}$, and L. Chen ${ }^{1}$ \\ ${ }^{1}$ Griffith School of Engineering, Griffith University, Gold Coast, Australia \\ (andrew.huang@griffith.edu.au, r.stewart@griffith.edu.au, 1.chen@griffith.edu.au )
}

\begin{abstract}
Manufacturing companies have strived to enhance managerial and technical capabilities to improve business performance. Building these capabilities requires effective share of knowledge - the strategic resource. Specifically, knowledge sharing (KS) between different manufacturing departments can improve manufacturing processes since leveraging organisational knowledge plays an enssential role in achieving competitive advantage. This paper presents an empirical investigation into the impact of KS on the effectiveness of supply chain management (SCM) and the product development process (PDP) in achieving desired business performance (BP). A questionnaire survey was administered from electronic manufacturing companies operating in Taiwan. 168 valid responses were received and used to statistically examine the relationships between the concepts (SCM, PDP, KS, BP). The study findings reveal that within the Taiwanese electronic manufacturing companies $\mathrm{KS}$ is an essential enabler for facilitating the effectiveness of SCM and PDP in achieving desired BP.
\end{abstract}

Keywords - Knowledge sharing, Supply chain management, Product development process, Business Performance

\section{INTRODUCTION}

In the face of highly competitive and expanding global marketplaces, manufacturing companies are seeking ways to sustain their competitiveness. Competitive advantage lies with those companies who can produce products of superior quality, reliability, flexibility and performance, while reducing costs and introducing the product to the market faster [1]. As many other developed countries, Taiwanese manufacturing companies are no exception. They are endeavouring to streamline the company in every, even subtle aspect of business activities and strategies, especially in product design and process improvement [8].

SCM becomes one of the most powerful business concepts for organisations to gain a competitive advantage in the global marketplace [2]. Companies who have achieved supply chain integration success report lower investments in inventory, reduced production cycle times, lower material acquisition costs, higher employee productivity and operational efficiency, increased ability to meet customer requirements and lower logistics costs [3]. A key point in SCM is that the entire process must be viewed as one system. In general, SCM seeks to improve the performance through elimination of waste and better leveraging of internal teamwork and external supplier capabilities and technologies [4].

PDP concerns about the implementation of various design activities which contribute to the effectiveness of new product design [5]. The impact of PDP is not only restricted to quality and cost dimensions; it can also significantly impact on production, process and business performance [6]. Market competition requires companies to procure and apply resources to create value by offering better products in a timely manner and with continuously improving efficiency. Therefore, companies emphasize faster and more efficient development processes, shorter and more cost effective design cycles and quicker delivery times [7]. In addition, effective product design and development performance has been recognised as an important market leadership tool by successful companies in competitive industries [8].

From most manufacturing industry perspectives, SCM and PDP activities require a high proportion of the company resources such as budget and manpower. Therefore, within manufacturing companies, SCM and PDP are two major business activities, which contribute to the companies' competitive advantage and performance $[9,10]$. In addition, KS is the key practice that enables SCM and PDP to achieve the desired performance more effectively $[10,11]$. The ability to communicate, capture, organise and disseminate knowledge allows companies to improve decision making, process efficiency, quality improvement, timeliness, customer satisfaction, and cost reduction [12]. It has been found that the winning companies must constantly create new knowledge through innovation. In the same time, they need to create synergy through sharing and harnessing internal knowledge and know-how as well as absorbing external knowledge and up-to-date technology [13]. Some researchers have asserted that the basic economic resource is no longer capital or natural resource but knowledge [12,13]. Therefore, knowledge based assets (e.g., intellectual capital) have become a primary form of organisational competence and the key to superior performance [10]. In other words, through reusing, refining and sharing information and knowledge, a company will improve business practices which will ultimately lead to advanced competitive advantage and performance [14].

Given the essential roles of SCM and PDP in manufacturing companies, a related issue that arises in 
this context is how KS can improve the SCM and PDP activities in terms of achieving desired performance more effectively and efficiently. As the empirical evidence for KS's moderation effect on the relationships between SCM, PDP and business performance (BP) is limited in the literature, the study reported by this paper attempted to shed some light on this issue. The study aims to provide answers to two major research questions: (1) Does the relationships exist between SCM, PDP and BP? (2) If the relationships do exist, does the intensity of KS moderate them? Based on the review of literature, four hypotheses (graphically illustrated in Fig. 1) were formulated to facilitate the investigation of the issues raised by the research questions:

- H1-1: SCM is positively associated with BP.

- H1-2: The higher the KS, the stronger the association of SCM with BP.

- H2-1: PDP is positively associated with BP.

- H2-2: The higher the KS, the stronger the association of PDP with BP.

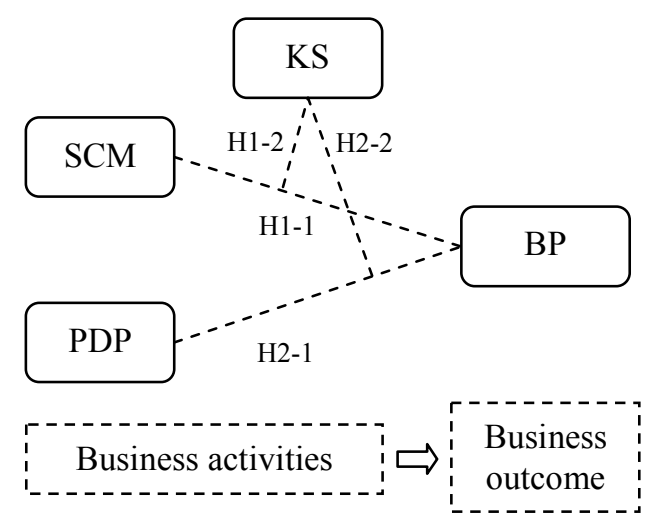

Fig. 1. Proposed conceptual model

The four concepts (SCM, PDP, KS, BP) of these hypotheses were operationalised based the previous empirical research studies [19-21].

\section{RESEARCH METHDOLOGY}

The research design mainly follows a deductive approach, which begins with an abstract, logical relationship between concepts, then moves toward concrete empirical evidence [15]. The purpose of this study is hypothesis testing, which offers a cross-sectional design to enhance understanding of the influence of KS activities on the effectiveness of SCM and PDP in achieving desired BP. The study was undetaken within the context of Taiwanese electronic manufacturing companies. A mailed questionnaire was chosen as the data collection method. The questionnaire was developed based on the operational definitions of the concepts and designed with two (2) major sections. Section 1 elicited respondents' opinions on the extent of SCM, PDP, KS activities were being executed by the companies, and the perceived BP level at the time of the survey. Five-point Likert scales were applied. Section 2 gathered demographic information about the respondents and their companies. The questionnaire was pre-tested with forty (40) managerial and professional staff members in Taiwan to evaluate the questionnaire for clarity, bias, ambiguous questions, and relevance to the business environment and operations of Taiwanese manufacturing companies. Thirty (30) respondents offered valid feedback and advice that was considered sufficient for serving the purpose [16]. The data collection process began after the questionnaire had been finalised, based on the pre-test feed back.

The research question statements indicate the unit of analysis is at the company level. Large- and mediumsized electronic manufacturing companies represent the theoretical population. To select sample candidates, companies representing the ' $\mathrm{C}$ ' industry (i.e. Communication, Computing, Consumer electronic, and Car electronic/automotive) listed in the Taiwan Stock Exchange (TSE) that were randomly drawn as potential study participants. During the survey process, the companies were first contacted via e-mail, telephone and networking to confirm contact details and to introduce the survey. The self-administered questionnaire were then mailed or delivered in person to the managerial and professional staff member(s) within these companies. A cover letter was attached to the questionnaire to introduce the researcher, objectives of the study, and the importance of the survey. A self-addressed, stamped return envelope was also included in the package. A total of 400 questionnaire packages were delivered. Telephone and email follow up were undertaken within a week subsequent to the delivery of the questionnaire packages, and necessary second mailing took place afterwards. More then 100 second mailing questionnaire packages were delivered.

\section{DATA ANALYSIS AND RESULTS}

\section{A. Data Preparation}

To provide statistical support for the research hypotheses, gathered data were analysed using a number of statistical techniques such as data examination, factor analysis and regression analysis processed through SPSS version 15.0 standard procedures. Over a four month period, useful responses were provided by 83 companies, which represents about $34.4 \%$ of the sampling frame. A total of 176 useful responses were received after completion of follow-up mailings. Since the subject matter is the respondents' companies, measured by the perception of the respondents, no more than five (5) useful feedback questionnaires were chosen from each company to avoid bias in the data. Based on this rule, 168 of 176 response questionnaires were retained for data 
analysis. The responses were considered a good representation of the opinions of the population, since the majority of the respondents were middle-aged, welleducated, experienced, and knowledgeable about manufacturing operations and management within their companies, as presented in Table I. Data screening techniques were applied to all variables to ensure that the data complies with the assumptions of normality and linearity $[17,18]$.

TABLE I

DEMOGRAPHIC INFORMATION OF RESPONDENTS

\begin{tabular}{rcc}
\hline Age & & Percentage \\
\hline $\begin{array}{r}\text { Demographic Information } \\
\text { More than 31 years }\end{array}$ & 122 & $72.6 \%$ \\
$\begin{array}{r}\text { Educational background } \\
\text { A bachelor degree or higher } \\
\text { Position }\end{array}$ & 139 & $82.7 \%$ \\
Executives & 31 & $18.5 \%$ \\
Managers & 66 & $39.2 \%$ \\
Senior engineers & 71 & $42.3 \%$ \\
industry experience & & \\
More than 4 years & 125 & $74.4 \%$ \\
Company operation year & & \\
More than 6 Years & 61 & $73.5 \%$ \\
Company categories & & \\
Product design and manufacturing & 60 & $72.3 \%$ \\
function & & \\
Product manufacturing function & 13 & $15.7 \%$ \\
Product design function & 10 & $12.0 \%$ \\
Company scale & & \\
Multinational & 61 & $73.5 \%$ \\
National and regional & 22 & $26.5 \%$ \\
No. of employee & & $14.5 \%$ \\
$\leq 200$ & 12 & $55.4 \%$ \\
201 - 500 & 46 & $12.1 \%$ \\
$>500$ & 15 & \\
Others & 10 & \\
\hline
\end{tabular}

\section{B. Scale Development}

The measurement variables were adopted from prior studies in the context of the manufacturing industry [1921]. As the number of scale items was reduced and descriptions modified, exploratory factor analysis was needed to assess the degree to which the data meet the expected structure. The 168 cases in the data file met the acceptable sample size of 100 for factor analysis; and was much larger than the minimum requirement of 80 , that was five (5) times as many subjects as the variables to be analysed in the concepts with the largest number of variables (16) [22].

As Table II presents, the factor analysis identified two (2) factors for the concept of SCM; two (2) factors for the concept of PDP; three (3) for the concept of KS, and four (4) factors for BP. The Kaiser-Meyer-Olkin (KMO) measure of sampling adequacy was greater than 0.60 , ranging from 0.61 to 0.71 , well above the acceptable level of 0.5 [22]. With the sample of 168, a factor loading of
0.50 and above was considered significant at 0.05 level to obtain a power level of $80 \%$ [22]. The cumulative percentage of total variance explained by the 11 extracted factors was greater than $50 \%$. Moreover, the reliability coefficient of all measures was above 0.70 , indicating good consistency of the scales for the concepts and their factors [22]. Since the concepts were conceptually defined based on a combination of the literature review, previous empirical studies, and the pilot study these scales were considered to have face validity [15], and they sufficiently measure the key practices of SCM, PDP, and KS and BP within the research context. These scales' nomological validity was confirmed by regression analysis, which is outlined in the following section [22].

TABLE II

RESULTS OF FACTOR ANALYSIS

\begin{tabular}{ccccc}
\hline Concept & $\begin{array}{c}\text { No. of } \\
\text { factors }\end{array}$ & $\begin{array}{c}\text { No. of } \\
\text { variables }\end{array}$ & $\begin{array}{c}\text { Cronbach's } \\
\text { Alpha }\end{array}$ & $\begin{array}{c}\text { Total Variance } \\
\text { Explained (\%) }\end{array}$ \\
\hline SCM & 2 & 7 & 0.81 & 71 \\
PDP & 2 & 6 & 0.74 & 69 \\
KS & 3 & 10 & 0.80 & 65 \\
BP & 4 & 11 & 0.78 & 74 \\
\hline
\end{tabular}

\section{Relationship Identification}

Correlation and regression analyses were employed in analysing the relationships between the concepts. Correlation analysis showed that the SCM, PDP and KS concepts were positively associated with the BP concept, with Pearson correlation $r$ (coefficient of correlation) values ranging from 0.598 to 0.302 (significant at 0.01 level). Simple regression analyses revealed that both SCM and PDP could be used to predict the variance of BP. Stepwise regression analyses with interaction term (SCM*KS and PDP*KS) were performed to investigate the respective effect of SCM and PDP on BP at different levels of KS (i.e., low, medium, high). Both the overall model validity ( $\mathrm{F}$ test) and the population correlation coefficient (t-test) of the derived regression models were significant at the 0.05 level [23]. In order to minimise multicollinearity, the independent variables (IVs) were centered and the $\mathrm{SCM}^{*} \mathrm{KS}$ and $\mathrm{PDP}^{*} \mathrm{KS}$ terms were formed by multiplying together the two centred terms [24]. The 168 cases in the data file satisfied the minimum sample size of 120 for supporting the case-to-IV ratio of 40 to 1 required by stepwise regression analysis with three IVs [25]. Multicollinearity was absent from the two selected models where tolerance values $(0.888$ and 0.753$)$ were much higher than 0.1 .

The simple regression models in Table III revealed that SCM and PDP could predict and explain the variance of $\mathrm{BP}$ by $35.3 \%$ and $8.6 \%$ percent respectively (with power of 0.80 ), whereby supporting hypotheses H1-1 and $\mathrm{H} 2-1$, that both SCM and PDP are positively associated 
with BP. Moreover, this finding also indicates a much stronger predicting power of SCM.

TABLE III

REGRESSION MODELS

\begin{tabular}{cccccc}
\hline Hypotheses & Significant regression models & $\mathrm{R}$ & $\mathrm{R}^{2}$ & $\begin{array}{c}\text { Adj. } \\
\mathrm{R}^{2}\end{array}$ & $\mathrm{~F}$ \\
\hline $\mathrm{H} 1-1$ & $\mathrm{BP}=19.889+0.552^{* *} \mathrm{SCM}$ & 0.598 & 0.357 & 0.353 & $92.20^{* *}$ \\
\hline $\mathrm{H} 1-2$ & $\mathrm{BP}=35.868+0.507 * * \mathrm{SCM}$ & 0.613 & 0.375 & 0.368 & $49.60^{* *}$ \\
& $+0.042 * \mathrm{SCM} \cdot \mathrm{KS}$ & & & & \\
\hline $\mathrm{H} 2-1$ & $\mathrm{BP}=25.542+0.336^{* *} \mathrm{PDP}$ & 0.302 & 0.091 & 0.086 & $16.66^{* *}$ \\
\hline $\mathrm{H} 2-2$ & $\mathrm{BP}=35.817+0.473^{* *} \mathrm{KS}$ & 0.601 & 0.361 & 0.353 & $46.64^{* *}$ \\
& $+0.058 * \mathrm{PDP} \cdot \mathrm{KS}$
\end{tabular}

Table III also reveals that in comparison with the simple regression models, the multiple regression models with interaction terms had larger predicting power over the variance of BP. In particular, the combination of KS and PDP*KS could explain $35.3 \%$ of the variance in BP, which was much larger than PDP could predict as a single IV.

Following Ref. [24], values of KS were chosen to be one standard deviation below the mean (KS low $=-3.04)$, at the mean (KS medium $=0.00)$, and one standard deviation above the mean $(\mathrm{KS}$ high $=3.04)$. Simple regression lines were then generated by substituting these values $(-3.04,0.00,3.04)$ in the two multiple regression models with the interaction terms in Table III. As the result of the computation, six simple regression equations were produced, and are illustrated in Fig. 2 and 3, where the impact of KS on the relationships between the two IVs (SCM or PDP) and BP are clearly revealed. The statistical significance of the slopes of these simple regression equations were also analysed according to Ref. [24].

The simple regression equations in Fig. 2 indicate a significant $(\alpha=0.05)$ positive regression of BP on SCM on all three levels of KS. The higher the KS level, the slightly steeper the slope. This suggests that KS has a positive, however not strong, impact on SCM's contribution to BP. Equations in Fig. 3 indicate a significant $(\alpha=0.01)$ positive regression of BP on PDP for the high KS level, no relationship between BP and PDP for the medium KS level, and a negative, but insignificant $(\alpha=0.05)$, relationship between BP and PDP at the low KS level. This finding reveals a critical role of $\mathrm{KS}$ in influencing the validity of PDP in terms of achieving desired performance. The regression analysis with interaction terms upholds the hypotheses H1-2 and $\mathrm{H} 2-2$, that the higher the KS, the stronger the positive association of SCM and PDP with BP.

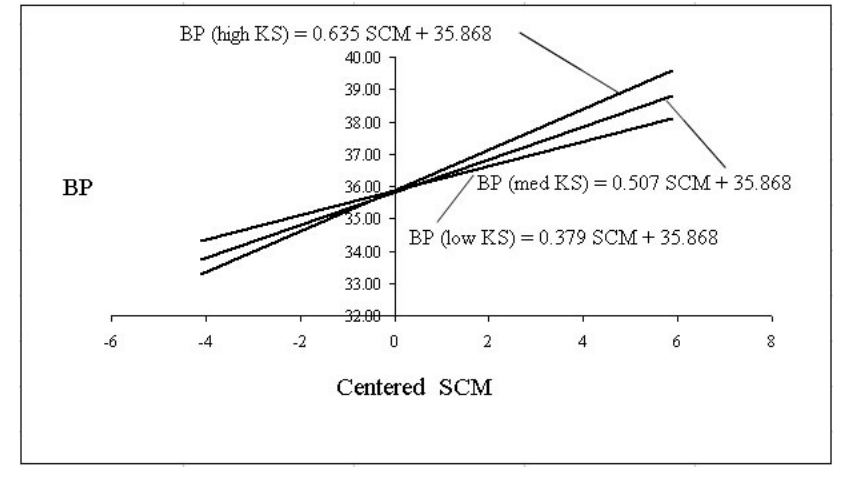

Fig. 2. Regression of BP on SCM at different level of KS

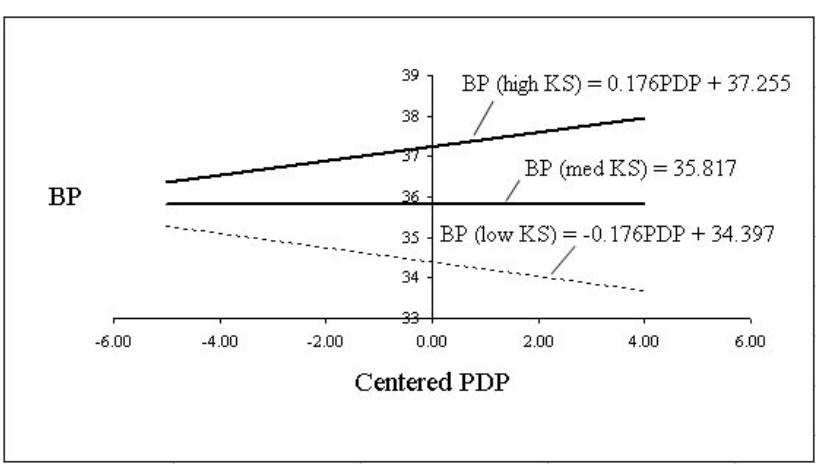

Fig. 3. Regression of BP on PDP at different level of KS

\section{CONCLUDING REMARKS}

The empirical findings reported herein reveal the moderation effect of KS upon the relationships between SCM, PDP and BP within the context of electronic manufacturing companies operating in Taiwan. These findings suggest that the ability of the manufacturing companies to absorb and share the needed knowledge from both internal and external sources has a significant impact on the effectiveness of SCM and PDP in helping companies to achieve the desired BP.

The study reveals a moderate influence of KS on the relationship between SCM and BP. With higher KS, companies are more likely to create better and innovative practices to manage the whole supply chain which can lead to improved business performance. Since most of these SCM practices are perceived as routine procedures within the manufacturing context, even when KS are performed at a medium or low level, SCM still has a slight positive impact on $\mathrm{BP}$ as long as the company follows the standard procedures to implement business practices.

On the contrary, PDP practices are primarily knowledge-intensive activities in manufacturing companies. Only through extensive KS within the company from the very early design stage, the PDP can make effective positive contributions to the BP. 
Otherwise, BP would not be easily improved through the PDP since mediocre KS would not create enough knowledge intensity to support these highly technical and creative design activities. Hence, it is essential for manufacturing companies to create a culture to facilitate KS. The study findings indicate that fostering a cooperative relationship internally and externally based on trust, communication, employee empowerment and commitment is critical to produce creative and high quality design which reflects the core competence of a manufacturing company.

It is worthwhile to note that the findings of this empirical study were derived from a sample of only one industrial sector (electronic manufacturing companies) from a specific geographical location (Taiwan). The generalisability of the findings could be increased by future studies based on larger samples of participants from diverse industry sectors and cultural backgrounds. Qualitative approaches such as in-depth case studies may also be helpful in providing further insights of the phenomenon under the investigation.

\section{REFERENCES}

[1] K. L. Choy, W. B. Lee, C. W. Lau, and L. C. Choy, "A knowledge-based supplier intelligence retrieval system for outsource manufacturing", Knowledge-Based System, vol. 18, pp. 1-17, 2005.

[2] D. M. Lambert and M. C. Cooper, "Issues in supply chain management", Industrial Marketing Management, vol. 29, pp. $65-83,2000$.

[3] R. R. Lummus and R. J. Vokurka, "Defining supply chain management: a historical perspective and practical guidelines", Industrial Management \& Data System, vol. 99, no. 1, pp. 11-17, 1999.

[4] N. O. Ndubisi, M. Jantan, L. C. Hing, and M. S. Ayub, "Supplier selection and management strategies and manufacturing flexibility", Enterprise Information Management, vol. 18, no. 3, pp. 330-349, 2005.

[5] P. Hong, M. A. Vonderembse, W. J. Doll, and A. Y. Nahm, "Role change of design engineers in product development", Operations Management, vol. 24, pp. 63-79, 2005.

[6] Y. Y. Lu and C. Yang, "The R\&D and marketing cooperation across new product development stages: an empirical study of Taiwan's IT industry", Industrial Marketing Management, vol. 33, pp. 593-605, 2004.

[7] C. L. Tan and M. A. Vonderembse, "Mediating effects of computer-aided design usage: from concurrent engineering to product development performance", Operations Management, vol. 24, pp. 494-510, 2006.

[8] S. L. Ahire and P. Dreyfus, "The impact of design management and process management on quality: an empirical investigation", Operations Management, vol. 18, pp. 549-575, 2000.

[9] K. C. Tan, V. R. Kannan, R. B. Handfield, and S. Ghosh, "Supply chain management: an empirical study of its impact on performance", Operations \& Production Management, vol. 19, no. 10, pp. 1034-1052, 1999.
[10] R. Lubit, "Tacit knowledge and knowledge management: The keys to sustainable competitive advantage", Organisational Dynamics, vol. 29, no. 4, pp. 164-178, 2001.

[11] S. B. Modi and V. A. Mabert, "Supplier development: Improving supplier performance through knowledge transfer", Operations Management, vol. 25, pp. 42-64, 2006.

[12] H. A. Artail, "Application of KM measures to the impact of a specialized groupware system on corporate productivity and operations", Information \& Management, vol. 43, pp. 551-564, 2006.

[13] V. Peltokorpi and E. Tsuyuki, "Knowledge governance in a Japanese project-based organization", Knowledge Management Research \& Practice, vol. 4, pp. 36-45, 2006.

[14] P. Ingram and T. Simons, "The transfer of experience in groups of organizations: implications for performance and competition", Management Science, vol. 48, no. 12, pp. 1517-1533, 2002.

[15] W. L. Neuman, "Social research methods: qualitative and quantitative approaches", Fifth Edition, Allyn and Bacon, 2003.

[16] A. C. Burns and R. F. Bush, "Marketing research", Prentice Hall, New Jersey, 1998.

[17] S. J. Coakes, "SPSS: analysis without anguish: version 12.0 for Windows", John Wiley \& Son Australia , Ltd, 2005.

[18] J. Pallant, "SPSS survival manual: a step by step guide to data analysis using SPSS for windows: version 10 for Windows", Allen \& Unwin, St Leonards, N.S.W., 2001.

[19] K.C. Tan, V. R. Kannar, R. B. Handfield, and S. Ghosh, "Supply chain management: an empirical study of its impact on performance", Operations \& Production Management, vol. 19, no. 10, pp. 1034-1052, 1999.

[20] K. J. Petersen, R. B. Handfield, and G. L. Ragatz, "Supplier integration into new product development: coordinating product, process and supply chain design", Operations Management, vol. 23, pp. 371-388, 2005.

[21] I. C. Hsu, "Enhancing employee tendencies to share knowledge - case studies of nine companies in Taiwan", Information Management, vol. 26, pp. 326-338, 2006.

[22] J. F. Hair, R. E. Anderson, R. L. Tatham, and W. C. Black, "Multivariate data analysis", Fifth Edition, Prentice-Hall International, Inc, Upper Saddle River, N. J., 1998.

[23] B. L. Bowerman, R. T. O'Connell, and D. A. Dickey, "Linear statistical models", Duxbury Press, Boston, 1986.

[24] L. S. Aiken, S. G. West, and R. R. Reno, "Multiple regression: testing and interpreting interactions", Newbury Park, Calif. : Sage Publications, 1991.

[25] B. G. Tabachinick, and L. S. Fidell, "Using multivariate statistics", Fourth Edition, Allyn and Bacon, 2001. 